\title{
Information-Theoretic Bounds for Mobile Ad-hoc Networks Routing Protocols
}

\author{
Nianjun Zhou and Alhussein A. Abouzeid * \\ Dept of Electrical, Computer and Systems Engineering \\ Rensselaer Polytechnic Institute \\ Troy, New York 12180, USA \\ email:zhoun@rpi.edu, abouza@rpi.edu
}

\begin{abstract}
In this paper, we define the routing overhead as the amount of information needed to describe the changes in a network topology. We derive a universal lower bound on the routing overhead in a mobile ad-hoc network. We also consider a prediction-based routing protocol that attempts to minimize the routing overhead by predicting the changes in the network topology from the previous mobility pattern of the nodes. We apply our approach to a mobile ad-hoc network that employs a dynamic clustering algorithm, and derive the optimal cluster size that minimizes the routing overhead, with and without mobility prediction. We believe that this work is a fundamental and essential step towards the rigorous modeling, design and performance comparisons of protocols for ad-hoc wireless networks by providing a universal reference performance curve against which the overhead of different routing protocols can be compared.
\end{abstract}

\section{Introduction}

Research on distributed multi-hop wireless networks, also known as wireless ad-hoc networks [1] has evolved from DARPA packet radio program during the early 1970's [2]. Since wireless ad-hoc networks can be deployed rapidly in a non-organized (i.e. ad-hoc) fashion without requiring any existing infrastructure, they are expected to find applications in a number of diverse settings [3,4].

Much of the research in the area of ad-hoc networks has focused on developing routing protocols. Proactive routing protocols (e.g. [5-7]) attempt to compute paths in advance and determine them continuously so that a route is readily available when a packet needs to be forwarded. Reactive routing protocols (e.g. [8-10]) are based on a source initiated query/reply process and typically rely on the flooding of queries for route discovery. A network with a few relatively fast moving nodes favors reactive protocols while a network with many slowly moving nodes favors proactive protocols.

The two strategies are combined in [11] which can be thought of a hierarchal (clustered) routing scheme. Hierarchical routing schemes based on the formation of a virtual backbone are developed in $[12,13]$. The main advantage of hierarchal routing is that it overcomes scalability problems by designating a node within a group of nodes to be

\footnotetext{
* Correspondence Author: Alhussein A. Abouzeid, Rensselaer Polytechnic Institute, 110 Eighth St, JEC-6038, Troy NY 12180 Tel: (518)276-6534, Fax: (518)276-4403
} 
responsible for routing, and thus only these nodes need to maintain routing information about the rest of the network. The main disadvantage is the overhead in the cluster maintenance operations (i.e. joining and leaving the clusters) which mainly depend on the degree of mobility of the nodes. This paper considers proactive hierarchal routing protocols.

Considerable effort has been directed towards evaluating routing protocols [14-21]. The procedure of evaluation relies on simulations of the protocol and comparing its performance against other existing protocols.

There is a need for a universal reference performance curve that can tell us how good (or bad) the performance of a specific protocol really is. In coding theory, a channel coding algorithm is good if it achieves the Shannon capacity [22]. Similarly, we seek to derive a universal curve against which we can measure how good a routing protocol performs, in terms of minimizing the routing overhead, which is the amount of information needed to describe the changes in a dynamic network topology. Up to our knowledge, this paper is the first that attempts to derive a theoretical lower bound on the routing overhead of ad-hoc network protocols. Related work in [23,24] do not consider the routing overhead in deriving the capacity of wireless networks.

\section{Network Model}

\subsection{Assumptions}

We make the following assumptions about the network: (A1) The mobile nodes are identical; (A2) The mobile nodes are distinguishable;(A3) The number of mobile nodes in the network is fixed, denoted by $N$; (A4) The communication region of interest is fixed and bounded. A1 means that all the nodes have the same physical characteristics. A2 means that each node is addressable by a node unique identifier - which we denote as $N U I$. Furthermore, We assume that the statistical node mobility patterns of individual nodes are independent and known. In this paper, we allow any type of mobility model as long as it allows a formulation using Markov chains.

Regarding the self-organization of the network and the geographic distribution of the nodes, the model can be viewed as a two-level abstraction. We assume that nodes move freely within a bounded region of space. The whole region is divided into subregions. For this preliminary work, we assume the number of sub-regions is fixed. There are two levels of hierarchy for nodes. Each region has its unique identifier - RUI. All the nodes of a sub-region form a cluster and a cluster head, which we call routing node. Routing node is selected at random from the nodes within the sub-region. It is possible that a sub-region becomes empty and thus will not have a cluster or a cluster head. The nodes belonging to the same sub-region communicate directly. The nodes belonging to different sub-regions communicate through the routing nodes. Thus, only two modes of direct (i.e. single-hop) communication are permissible: nodes within the same subregion, and routing nodes of neighboring sub-regions (See Figure 1).

\subsection{Definitions}

For clarity of presentation, we provide the formal definition of the terms used in the paper as follows. Routing Node: The cluster head for a sub-region, assumed to be 


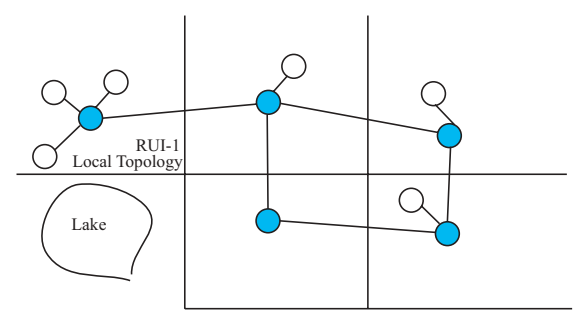

Fig. 1. A snapshot of the network topology. Blue (dark) nodes are routing nodes. There is one routing node in every sub-region. Sub-regions do not have to be rectangular or identical.

selected randomly from the set of nodes that belong to the sub-region. There is only one routing node for a sub-region. If a routing node leaves a sub-region, a new routing node is selected from the remaining nodes in the same sub-region. Each routing node has an RUI associated with it; Regular Node: a node that is not currently a routing node. Any regular node can be selected as a routing node; Topology: is the connectivity relationship of the nodes as depicted in Figure 1. Due to the dynamic behavior of the topology, it can be described as an instance of a random graph. An edge exists between two arbitrary nodes if single-hop communication between those two nodes is admissible, according to the rules presented earlier. Due to the mobility of the nodes, the edge of two nodes can be established or torn down randomly; Global Topology: The topology of the whole network. Local Topology: The topology of a sub-region; Topologies Cardinality: The total number of the topologies. The cardinality of the network topologies is used to calculate the minimum amount of information needed to describe a network snapshot without any extra knowledge; Information overhead: The bits needed to distinguish one topology from another.

\section{Analysis}

Any algorithm for solving some problem must do some minimal amount of work. The most useful principle of this kind is that the outcome of a comparison between two items contains at most one bit of information. Hence, if there are $m$ possible input strings, and an algorithm purports to identify which one it was given solely on the basis of comparisons between input symbols, then $\left\lceil\log _{2} m\right\rceil$ comparisons are needed (all logarithms in this paper are base 2 and hence we will shortly write log instead of $\left.\log _{2}\right)$. This is because $\lceil\log m\rceil$ bits are necessary to specify one of the $m$ possibilities [25].

\subsection{Outline and notation}

In this section, we apply the above principle to derive a lower bound on the overhead of proactive hierarchal routing protocols with fixed number of clusters in a mobile wireless ad-hoc network. The steps followed are as follows: (a) Compute the number of different topologies possible for each local sub-region and for the whole network. These are 
termed local topologies cardinality and global topologies cardinality, and are denoted by denoted $\mathbf{L}$ and $\mathbf{G}$, respectively; (b) Derive expressions for $h$, the average holding time for a node within a sub-region, and for $H$, the average holding time before a topology change; (c) Compute the minimum amount of information needed to keep track of the topology changes using the individual local information from the different sub-regions $\mathbf{I}_{\mathbf{L}}$ and from the global view of the network $\mathbf{I}_{\mathbf{G}}$. (d) Finally, consider the effect of predicting the future locations of the nodes based on their mobility on the local $\mathbf{I}_{\mathbf{L}}^{\mathbf{P}}$ and global $\mathbf{I}_{\mathrm{G}}^{\mathbf{P}}$ routing overhead.

\subsection{Cardinalities of Global and Local Network Topologies}

Theorem 1: Consider $N$ mobile nodes randomly distributed over $M$ sub-regions. Choose a routing node for each non-empty sub-region randomly from the set of nodes belonging to the sub-region. The total number of possible topologies is given by:

$$
G=\sum_{k=1}^{\min (N, M)} \frac{N ! M !}{k !(M-k) !(N-k) !} k^{N-k}
$$

Proof $^{1}:$ See Appendix.

Theorem 2: Consider $N$ mobile nodes randomly distributed over $M$ sub-regions, then the total number of local topologies for a given sub-region is:

$$
L=\left(2^{N-1}\right) N+1
$$

Proof: See Appendix.

\subsection{Inferring Network Topology Dynamics from Individual node Mobility}

We consider the following simple Markov mobility model. For a given time-step $\tau$, we assume that ${ }^{2}$ the probability that a mobile node will stay in the same sub-region is $0 \leq p_{0} \leq 1$, and leave its current sub-region is $1-p_{0}$. We further assume that a node moves to any of the $K$ neighboring nodes with equal probability (random walk) $\frac{1-p_{0}}{k}$. Thus, the probability distribution function of the node holding time at the same sub-region is geometric. For a given node, the mean holding time $h=\frac{p_{0}}{1-p_{0}}$.

Since the individual nodes move independently, The probability $p_{1}$ that a specific topology does not change is equal to the probability that all nodes do not change their sub-regions. Assuming independent node mobility, $p_{1}=p_{0}^{N}$. Hence, the average holding time for a topology is thus

$$
H=\sum_{i=1}^{\infty} i\left(1-p_{1}\right) p_{1}^{i}=\frac{p_{1}}{1-p_{1}^{2}}=\frac{p_{0}^{N}}{1-p_{0}^{N}}
$$

\footnotetext{
${ }^{1}$ The result can be verified using a simple example with $N=4$ and $M=2$, where it is easy to show using manual calculation that the total combination is 56 , which is the same as the result from (1).

2 This model follows from discretizing a continuous-time two-dimensional Markov process description of the individual node mobility.
} 


\subsection{Routing Overhead without Mobility Prediction}

The information needed to identify a specific network topology from the set of all the possible topologies is the entropy of the set.

\section{Global Topology without Prediction: From (1)}

$$
I_{G}=\log (G)
$$

Since we do not have any knowledge of the topology probability distribution, we use the maximum entropy method [26] to infer it. From the proof of Theorem 1, we have shown that for a distribution $r=\left(r_{1}, r_{2}, \ldots, r_{M}\right)$ of nodes for each sub-region, the total number of possible topologies is

$$
n(r, N)=\frac{N !}{r_{1} ! r_{2} ! \ldots r_{M} !} \prod_{i=1}^{M} g\left(r_{i}\right) \text { with } \sum_{i=1}^{M} r_{i}=N
$$

We have also shown that $\prod_{i=1}^{M} g\left(r_{i}\right)$ reaches its maximum value when $r=\left(r_{1}, r_{2}, \ldots, r_{M}\right)$ has a uniform distribution. In this case

$$
n(r, N) \leq \frac{N !}{r_{1} ! r_{2} ! \ldots r_{M} !}\left\lceil\frac{N}{M}\right\rceil^{M}
$$

It is easy to show after some algebraic manipulations that

$$
M^{N} \leq G \leq\left\lceil\frac{N}{M}\right\rceil^{M} M^{N}
$$

and equivalently

$$
N \log M \leq I_{G} \leq N \log M+M \log \left\lceil\frac{N}{M}\right\rceil
$$

The result (8) can be interpreted as follows. The lower bound in (8) is the minimum information needed to describe the network without identifying which nodes are the routing nodes. The introduction of routing nodes increases the complexity of the network by $M \log \left\lceil\frac{N}{M}\right\rceil$.

Local Topology without Prediction: The entropy of a specific sub-region is $\log L$. From (2), the sum of the information overhead required to maintain each of the local topologies, $I_{L}$, is ,

$$
I_{L}=M \log \left(2^{N-1} N+1\right)
$$

where the " $M$ " coefficient comes from the fact that there are $M$ local topologies.

For large $N$,

$$
\lim _{N \rightarrow \infty} \frac{\log \left(2^{N-1} N+1\right)}{N}=1
$$

and hence

$$
I_{L} \approx M N ; N \gg 1
$$


From (8) and (11),

$$
\frac{I_{L}}{I_{G}} \approx \frac{M}{\log M} ; N \gg 1
$$

A comment on the above result (12) is in order. It states that, in the limit $N \rightarrow \infty$, $I_{L}>I_{G}$ (i.e. the sum of the entropies of the local topologies is larger than the entropy computed directly from the global network topology). The reason is that $I_{G}$ assumes the knowledge of $N$ and $M$ when computing the different possible topologies, while $I_{L}$ computes the local topology information locally (independent of the node distribution over the rest of the sub-regions) even though the local topologies of different sub-regions are not independent (a node may not exist in two different sub-regions).

\subsection{Routing Overhead with Mobility Prediction}

Global topology with prediction: The information needed to update the new location of a node (i.e. which sub-region it belongs to) given the knowledge of the current location is

$$
I=-1\left(p_{0} \log p_{0}+\left(1-p_{0}\right) \log \left(\frac{1-p_{0}}{k}\right)\right)
$$

and hence

$$
I_{G}^{P} \leq-N\left(p_{0} \log p_{0}+\left(1-p_{0}\right) \log \left(\frac{1-p_{0}}{k}\right)\right)+M \log \left\lceil\frac{N}{M}\right\rceil
$$

From (8) and (14),

$$
\frac{I_{G}^{P}}{I_{G}} \leq \frac{\log K+1}{\log M} ; N>>1
$$

For many practical scenarios, even the largest number of neighboring sub-regions is usually less than the total number of sub-regions. In this case, for large $N$, the above result states that using prediction to update the topology information will result in large savings in the routing overhead.

Local Topology with prediction: Assuming each node is equally likely to belong to any of the sub-regions with probability $\frac{1}{M}$,

$$
I_{L}^{P} \leq-M N\left(\frac{1}{M} \log \frac{1}{M}+\left(1-\frac{1}{M}\right) \log \left(1-\frac{1}{M}\right)\right)+M \log N
$$

Note that $M \log N$ bits are needed to specify the routing node for the cluster.

For large $M$,

$$
I_{L}^{P} \leq N \log M+M \log N ; M \gg 1
$$

From (11) and (17),

$$
\frac{I_{L}^{P}}{I_{L}} \approx \frac{N \log M+M \log N}{N M} ; M \gg 1 ; N \gg 1
$$




\section{Numerical Results and Applications}

In this section, we show some numerical results from the bounds derived in the previous section as well as an application of these bounds in finding the optimal number of subregions that minimizes the information overhead.

Figure 2 shows a numerical example for the lower bound on the routing overhead with and without prediction. Clearly, large amount of savings is achieved with prediction.

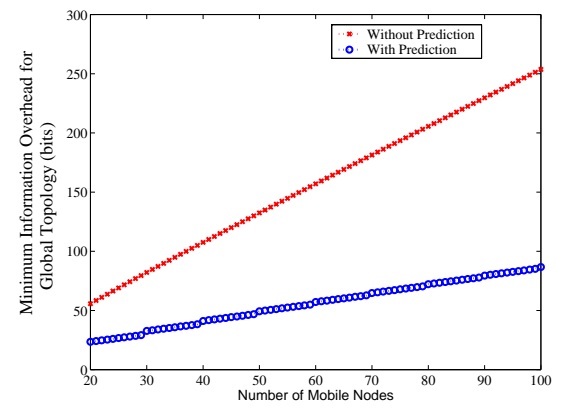

Fig. 2. Minimum information overhead with and without prediction. $M=5, p_{0}=$ $0.9, k=3$.

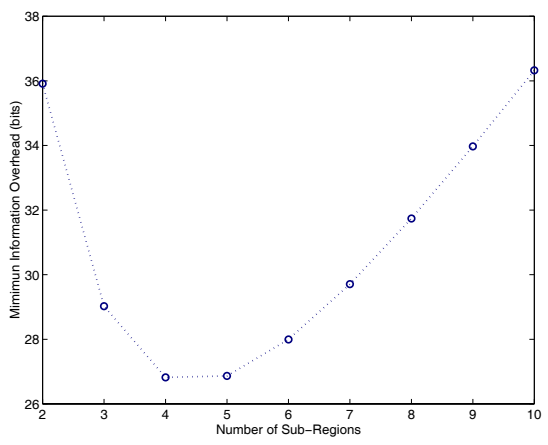

Fig. 3. Minimum information overhead, with prediction. $N=100, q=0.5, p_{0}=$ 0.9 and $k=3$. Optimal number of subregions is 4 .

Let $q$ denote the probability that two arbitrary communicating nodes belong to the same cluster. Let $O\left(O^{P}\right)$ denote the minimum routing overhead without(with) prediction. Then,

$$
O=q \frac{I_{L}}{M}+(1-q) I_{G}
$$

without prediction and

$$
O^{P}=q \frac{I_{L}^{P}}{M}+(1-q) I_{G}^{P}
$$

Figure 3 depicts the results for an arbitrary network. The figure shows the optimal number of sub-regions which is the one that requires the least amount of information exchange. Decreasing the number of sub-regions $M$ increases the information overhead in maintaining the local cluster topology (more nodes per cluster) while increasing the number of sub-regions increases the overhead in maintaining the inter-cluster (global) topology, and the optimal point represents the balance between those two opposing factors. 


\section{Conclusion}

This paper presented an information-theoretic approach to the analysis of routing protocols. We applied the framework to derive lower bounds on the routing overhead in a mobile ad-hoc network, with and without mobility prediction. We quantified the amount of overhead needed for clustering, and also the amount of savings. By combining both, we find the optimum number of clusters that minimize the routing overhead. A number of future avenues of work remain. Due to the distributed nature of the network, the local topology information will be exchanged and used to infer the global network topology. Therefore, the mechanism in which the local topology information will be exchanged is an important topic that has not been addressed in this paper. Including this aspect may result in a tighter lower bound (i.e. a curve that is "above" the one derived in this paper).

\section{References}

1. Z.J. Haas et al. eds. Wireless ad hoc networks. IEEE Journal on Selected Areas in Communications, 17(8), August 1999.

2. J. Jubin and J. D. Tornow. The DARPA packet radio network protocols. Proceedings of the IEEE, 75(1), 1987.

3. D. L. Estrin et al. Embedded Everywhere: A research agenda for networked systems of embedded computers. National Research Council, 2001. http://www.nap.edu.

4. I. F. Akyildiz, W. Su, Y. Sankarasubramaniam, and E. Cayirci. Wireless sensor networks: A survey. Computer Networks (Elsevier), 38(4):393-422, March 2002.

5. C. E. Perkins and P. Bhagwat. Highly dynamic Destination-Sequenced Distance-Vector routing (DSDV) for mobile computers. In Proceedings of ACM SIGCOMM'94, pages 234-44, London, United Kingdom, October 1994.

6. P. Jacquet, P. Muhlethaler, and A. Qayyum. Optimized link state routing protocol. IETF MANET, Internet Draft, November 1998.

7. S. Murthy and J. J. Garcia-Luna-Aceves. A routing protocol for packet radio networks. In Proceedings of ACM International Conference on Mobile Computing and Networking (MOBICOM '95), pages 86-94, Berkeley, CA, USA, December 1995.

8. V. D. Park and M. S. Corson. A highly adaptive distributed routing algorithm for mobile wireless networks. In Proceedings of IEEE INFOCOM'97, volume 3, pages 1405-13, Kobe, Japan, April 1997.

9. D. B. Johnson and D. A. Maltz. Dynamic source routing in ad hoc wireless networking. In T. Imielinski and H. Korth, editors, Mobile Computing. Kluwer, 1996.

10. C. E. Perkins and E. M. Royer. Ad hoc on-demand distance vector routing. In Proceedings of IEEE Workshop on Mobile Computing Systems and Applications (WMCSA'99), pages 90100, New Orleans, LA, USA, February 1999.

11. M. R. Pearlman and Z. J. Haas. Determining the optimal configuration for the zone routing protocol. IEEE Journal on Selected Areas in Communications, 17(8):1395-414, August 1999.

12. B. Das, R. Sivakumar, , and V. Bharghavan. Routing in ad hoc networks using a spine. In Proceedings of International Conference on Computer Communications and Networks, pages 34-9, Las Vegas, NV, USA, September 1997.

13. B. Das, R. Sivakumar, , and V. Bharghavan. Routing in ad-hoc networks using minimum connected dominating sets. In Proceedings of International Conference on Communications (ICC'97), volume 2, pages 765-9, Montreal, Canada, September 1997. 
14. S. Ramanathan and M. Steenstrup. A survey of routing techniques for mobile communications networks. Journal of Special Topics in Mobile Networks and Applications (MONET), 1(2):89-104, October 1996.

15. E. Royer and C.-K. Toh. A review of current routing protocols for ad-hoc mobile wireless networks. IEEE Personal Communications Magazine, pages 46-55, April 1999.

16. J.-C. Cano and P. Manzoni. A performance comparison of energy consumption for mobile ad hoc network routing protocols. In Proceedings of 8th International Symposium on Modeling, Analysis and Simulation of Computer and Telecommunication Systems (MASCOTS), pages 57-64, San Francisco, CA, USA, August 2000.

17. S.R. Das, R. Castaneda, and Yan Jiangtao. Simulation-based performance evaluation of routing protocols for mobile ad hoc networks. Mobile Networks and Applications, 5(3):17989, 2000.

18. C. E. Perkins, E. M. Royer, S. R. Das, and M. K. Marina. Performance comparison of two ondemand routing protocols for ad hoc networks. IEEE Personal Communications, 8(1):16-28, February 2001.

19. A. Boukerche. A simulation based study of on-demand routing protocols for ad hoc wireless networks. In Proceedings of IEEE 34th Annual Simulation Symposium, pages 85-92, Seattle, WA, USA, April 2001.

20. C.-K. Toh, H. Cobb, and D.A. Scott. Performance evaluation of battery-life-aware routing schemes for wireless ad hoc networks. In Proceedings of IEEE International Conference on Communications (ICC), volume 9, pages 2824-9, Helsinki, Finland, June 2001.

21. A. Nasipuri, R. Burleson, B. Hughes, and J. Roberts. Performance of a hybrid routing protocol for mobile ad hoc networks. In Proceedings Tenth International Conference on Computer Communications and Networks, pages 296-302, Scottsdale, AZ, USA, October 2001.

22. C.E. Shannon. A mathematical theory of communication (2 parts). Bell System Technical Journal, 27:379-423, 623-56, July and October 1948. http://cm.belllabs.com/cm/ms/what/shannonday/shannon1948.pdf.

23. P. Gupta and P.R. Kumar. The capacity of wireless networks. IEEE Transactions on Information Theory, 46(2):388-404, March 2000.

24. M. Grossglauser and J. Bolot. On the relevance of long range dependence in network traffic. IEEE/ACM Transactions on Networking, 7(5):629-40, October 1999.

25. B. Weide. A survey of analysis techniques for discrete algorithms. ACM Computing Surveys, 9(4):291-313, December 1977.

26. A. Papoulis and S.U. Pillai. Probability, Random Variables and Stochastic Processes. Cambrige studies in advanced mathematics. McGraw-Hill, 4th. edition, 2002.

\section{Appendix: Proof of theorems}

Proof of Theorem1 First, consider the case $N \geq M$. Let $r=\left(r_{1}, r_{2}, \ldots, r_{M}\right)$ denote a specific organization of the nodes over the sub-regions. Then the possible topologies for this case is

$$
n(r, N)=\frac{N !}{r_{1} ! r_{2} ! \ldots r_{M} !} \prod_{i=1}^{M} g\left(r_{i}\right)
$$

where $g(r)=r \forall r>0$ and $g(0)=1$. The function $g(r)$ follows from the fact that we have $r$ different ways of selecting a routing node for each subregion. 
Let $R$ denote the set of all possible organizations of the nodes. Let

$$
f\left(x_{1}, x_{2}, \ldots, x_{M}\right)=\left(x_{1}+x_{2}+\cdots+x_{M}\right)^{N}=\sum_{r \in R} \frac{N !}{r_{1} ! r_{2} ! \ldots r_{M} !} x_{1}^{r_{1}} x_{2}^{r_{2}} \ldots x_{M}^{r_{M}}
$$

The total number of topologies in which there are no empty sub-regions can be calculated by taking the partial derivative of (21) w.r.t $x_{i}$ and then setting $x_{i}=1$, which yields

$$
\left(\frac{N !}{(N-M) !}\right) M^{N-M}
$$

Consider now that there is a single empty sub-region $s$. Then the total number of topologies can be calculated by taking the partial derivative of (21) w.r.t $x_{i}$ and then set $x_{i}=1 \forall i \neq s$ and $x_{s}=0$, which yields

$$
\left(\frac{N !}{(N-M+1) !}\right) M^{N-M+1}
$$

And since there are $\left(\begin{array}{c}M \\ 1\end{array}\right)$ ways of having a single empty sub-region, the total number of topologies with a single sub-region is thus

$$
\left(\frac{M !}{1 !(M-1) !}\right)\left(\frac{N !}{(N-M+1) !}\right) M^{N-M+1}
$$

By induction, the number of topologies with exactly $i$ empty sub-regions is

$$
\left(\frac{M !}{i !(M-i) !}\right)\left(\frac{N !}{(N-M+i) !}\right) M^{N-M+i} ; 0 \leq i \leq M-1
$$

Summing over all $i$ yields the result. Similar derivation applies for the case $N<M$ (but notice in this case that the number of empty sub-regions will range from $M-N$ to $M-1)$.

Proof of Theorem2 Let $r$ be the number of nodes in the sub-region. The total number of possible topologies is thus

$$
L=\sum_{r=0}^{N} \frac{N !}{r !(N-r) !} g(r)
$$

where $g(r)$ has been defined in the proof of Theorem 1 (above). Let

$$
f\left(x_{1}, x_{2}\right)=\left(x_{1}+x_{2}\right)^{N}=\sum_{r=0}^{N} \frac{N !}{r !(N-r) !} x_{1}^{r} x_{2}^{N-r}
$$

Taking the partial derivative of both sides w.r.t $x_{1}$, then setting $x_{1}=x_{2}=1$ yields

$$
N 2^{N-1}=\sum_{r=1}^{N} \frac{N !}{r !(N-r) !} g(r)
$$

For the trivial case $r=0$ (no nodes in the sub-region, there is only one possible topology. 